Vol.9, No 1. Diciembre de 2017 pp. $169-181$

Artículo Original

Recibido para publicación: Septiembre 18 de 2017.

Aceptado para publicación: Noviembre 11 de 2017

\title{
Algunos apuntes sobre el acuerdo Comercial Unión Europea- Colombia: Un Bloque Económico contra una Nación.
}

\section{Some notes on the European Union-Colombia Trade Agreement: An Economic Block against a Nation.}

Autores: Yenis Barrios Arroyabe ${ }^{1}$

Yenny Margarita Mejía Turizo ${ }^{2}$

Correspondencia: ymbarriosa@hotmail.com, mejiaturizoyenny@gmail.com

\section{RESUMEN}

En el presente artículo reflexivo se pretende describir, comprender y analizar cómo son las relaciones comerciales entre Colombia y la Unión Europea, identificar cuáles serían las ventajas y desventajas a la hora de emprender dichas relaciones comerciales, siendo que Colombia es un exportador de materias primas y de allí su dependencia al comportamiento de la tasa de cambio y a su volatilidad, podrá ser una fortaleza o dificultad a la hora de la internacionalización del mercado.

En el presente artículo se pretende plantear los alcances que existen entre el TLC Colombia - Unión Europea y cómo puede afectar estas relaciones bilaterales la economía de la Región Caribe Colombiana, como área estratégica para la internacionalización de sus mercados. Se sabe que Colombia es un país que se encuentra territorialmente estratégico, situación que lo convierte en la mira de aperturas económicas, y este es un momento crucial ya que los aliados internacionales son necesarios para incentivar y fortalecer la exportación y diversificar los destinos, aumentar la inversión extranjera y obtener mayores accesos a tecnologías de punta, estos son puntos esenciales que se pretende con la firma del TLC entre Colombia y la Unión Europea.

\section{Palabras Claves:}

Competitividad, Acuerdos Comerciales, economía estable y sostenible.

\footnotetext{
${ }^{1}$ Contador público, especialista en gerencia financiera de la universidad libre, Diplomado en docencia universitaria.

2 Profesional en finanzas y relaciones internacionales de la Corporación Universitaria de la Costa CUC, especialista en gerencia financiera de la Universidad Libre de Barranquilla. Diplomado en negocios internacionales de la CUC
} 


\begin{abstract}
In this article it aims to describe, understand and analyze how would trade relations between Colombia and the European Union, to identify what would be the advantages and disadvantages when undertaking such business relationships, being that Colombia is an exporter of raw materials and hence its dependence on the behavior of the exchange rate and its volatility can be a strength or difficulty of internationalization of the market.

In this article it aims to raise the scope between Colombia FTA - European Union and how it can affect these bilateral relations in the economy of the Colombian Caribbean region as a strategic area for the internationalization of their markets. It is known that Colombia is a country that is geographically strategic position makes it the target of economic openings, and this is a crucial moment because international allies are needed to encourage and strengthen export and diversify destinations, increase foreign investment and obtain greater access to technologies, these are essential points that intended by the FTA between Colombia and the European Union.
\end{abstract}

\title{
Keywords:
}

Competitiveness, Trade Agreements, stable and sustainable economy.

\section{Introducción}

Actualmente el mundo está cambiando constante y aceleradamente y tiende a fuertes llamados comerciales a nivel internacional, tal es así que Colombia se ha transformado en los últimos años en una de las posibles potencias más llamativas de Latinoamérica, es por esto que el gobierno Colombiano al sentirse capaz de superar las expectativas del mercado internacional ha decidido integrar a Colombia en el mundo comercial, implementando unas políticas y estrategias de comercialización internacional que permitan tener acceso a todos los mercados incluyendo los de la Unión Europea. Cabe resaltar que Colombia siempre ha 
mantenido buenas relaciones comerciales con los países de la Unión Europea, siendo uno de los principales para exportar.

Estas relaciones comerciales buscan garantizar estabilidad, además de otorgar unos beneficios, y así la mayor generación de empleos posibles (Vanguardia, 2012), el crecimiento de una economía sostenible basada en la protección y promoción del trabajo y de los derechos ambientales en toda Colombia y la Región Caribe, logrando mantener una economía estable y sostenible.

\section{Metodología.}

El presente artículo se realizó a partir de una investigación de Corte descriptivo, con un enfoque mixto y con una orientación desde el punto de vista del método inductivo. El paradigma investigativo que se ajustó a los objetivos propuestos fue el crítico social. Las técnicas de recolección de información fueron esencialmente el análisis documental de fuentes secundarias.

\section{Resultados y discusiones.}

\section{Aspectos problemáticos entorno a los acuerdos comerciales de Colombia.}

Debido a la crisis europea entre finales de los años 2008 y 2009 la actividad comercial se debilitó, generando inestabilidad en el mercado europeo, es por esto que se ven en la obligación de abrirse a intercambios comerciales con países emergentes. 
En concordancia, Colombia está entre los países más cerrados del continente, aunque su crecimiento demuestre lo contrario, ya que la economía colombiana no está entre las más abiertas de Suramérica, como si lo es el caso chileno, es por ello que el gobierno está en la necesidad de la búsqueda de nuevas relaciones comerciales y aprovechar el dinamismo de esta, a través de tratados o acuerdos bilaterales. De esta forma, se observa que la Región Caribe es el principal puente estratégico para exportaciones e importaciones del mercado mundial, es por ello que se crea la necesidad de afrontar esta situación e identificar cómo afecta el impacto del mencionado TLC, reconociendo sus ventajas y desventajas para el desarrollo de una economía regional sostenible.

\section{Marco Jurídico}

Después de un análisis sopesado de la conveniencia de un posible Convenio Comercial entre el Gobierno Colombiano y el Bloque de la Unión Europea, teniendo en cuenta la gran afluencia de negociaciones con algunos de los países miembros. Este proceso de negociaciones fue un hecho que se consolidó con el acuerdo suscrito o firmado entre las partes en Bruselas, Bélgica el día 26 de Junio 2012, hecho que se generó con el fin de facilitar sus relaciones bilaterales que se encuentran fundamentadas en un marco de diálogo político y de cooperación, creando un marco jurídico el cual aumente la estabilidad en las relaciones de sus economías, otorgando beneficios (Güesguán, 2015). Posteriormente se aprueba este tratado a través de la Ley 1669 de 16 de Julio de 2013, quedando así instaurada dentro de los términos y las normas establecidas en este contrato, el cual rige a partir de la presente Ley, señalando las ventajas competitivas y comparativas en los productos exportados entre éstos. Dichas ventajas se pronuncian en la reducción de barreras arancelarias, lo que facilita la introducción de los productos en el mercado Europeo y Colombiano, facilitando de esta manera normas técnicas, como medidas sanitarias y fitosanitarias, derechos laborales y derechos humanos, inversiones, propiedad intelectual, el medio ambiente y demás 
factores que se adaptan en las reformas en las economías involucradas, teniendo como base el respeto hacía estas.

El principal objetivo de este acuerdo es establecer los estándares internacionales para garantizar el establecimiento y acceso al mercado para las inversiones en los sectores de bienes y de servicios. Además, se trataron disciplinas que tienden a asegurar un trato nacional justo y equitativo a las diferentes Compañías nacionales e inversionistas de los países negociados, dando por hecho un tratamiento no discriminatorio, pero tampoco menos favorable entre las partes y los involucrados.

\section{Análisis Estructural del mercado en Colombia}

Como bien se sabe, cabe recordar que Colombia inicio su apertura económica en los años 90, respuesta impulsada por la globalización de la economía mundial ya iniciadas en diferentes economías, dinámica de comercio que ha influido en Colombia la búsqueda de nuevos mercados.

El historial de relaciones que ha tenido Colombia en sus importaciones y exportaciones de bienes y servicios se muestra a continuación durante su apertura económica iniciada en los años 95 hasta el año 2010, lo que ha generado confianza en nuestra economía.

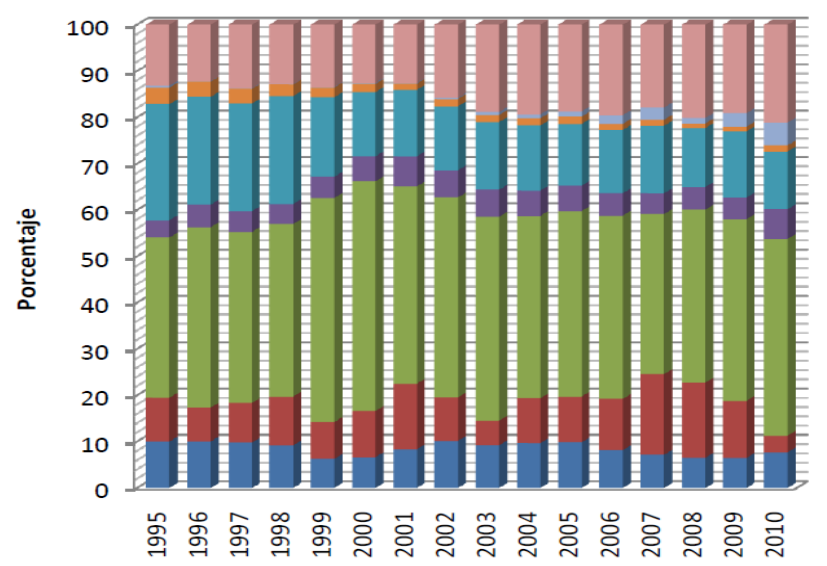

Año

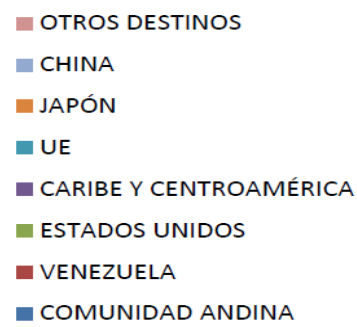


Fuente: Cálculos propios con datos del Ministerio de Comercio, Industria y Turismo (2016)

Como se puede observar en la gráfica anterior el índice y los principales países destinos de exportación de Colombia, se ve la necesidad de seguir abriendo puertas en el mercado Internacional. De esta manera Colombia le apuesta firmemente al proceso de internacionalización teniendo en cuenta la base de reglas claras, estables y predecibles que gobiernen el comercio internacional, resaltando su gran necesidad en mejorar el acceso a los diferentes destinos de exportación, ya que es importante la negociación y suscripción de nuevos acuerdos de libre comercio, el cual permita ser diverso el destino de las exportaciones y contribuir al incremento de la oferta exportable.

Tanto es así, que se vio en la necesidad de generar como se dice anteriormente nuevos mercados internacionales, y que mejor que con un Bloque a nivel Mundial de los más estables (UNIÓN EUROPEA), a pesar que sus relaciones con los Estados Unidos (Jetter, 2011) ha sido el mayor logro que ha obtenido Colombia, es más que claro que se quiere diversificar en el mercado Mundial, siendo Colombia el único país Latinoamericano que se encuentra geográficamente estratégico al estar rodeado de los dos océanos.

\section{Análisis de los Mercados: Relaciones Comerciales Colombia y la Unión Europea}

Para el estudio es imprescindible comenzar por abordar el análisis de la estructura del comercio internacional de Colombia y la Unión Europea, observando el comportamiento de las exportaciones e importaciones, del cual se puede examinar los sectores que se operan con mayor dinamismo. Es así, como se detendría a analizar la evolución de la internacionalización y/o globalización de los mercados en estos países, e identificar los sectores fuertes en cada uno de ellos. Un tratado de libre comercio (TLC) es el convenio mercantil obligatorio que firman dos o más estados para convenir el otorgamiento de preferencias arancelarias 
recíprocas y la disminución de barreras no arancelarias al comercio de bienes y servicios.

Actualmente, Colombia cuenta con ciertas operaciones comerciales, económicas y financieras que cada vez son más notorias a nivel internacional, y que aumentan nuestras expectativas comerciales.

Por su parte, la Unión Europea es una comunidad constituida por veintiocho (28) Estados europeos con el único fin de propiciar y generar una integración y gobernanza común de los estados y pueblos de Europa; consiguiendo de esta manera ser una potencia comercial (Wilhelmina et al, 2012). En general y económicamente la UE basa su crecimiento comercial en el sector de transporte, de la energía y de la investigación teniendo un cuidado extremo en no afectar el medio ambiente.

De los países que conforman este importante bloque económico, proveniente del viejo continente y que han mantenido relaciones económicas con Colombia, se encuentran: España, Países Bajos, Dinamarca, Alemania, Francia, entre otros; de los cuales Francia es el país que más se ha destacado, puesto que fue uno de los que más invirtió en la economía de nuestro país con aproximadamente 250 millones de dólares.

Es evidente que las relaciones entre Colombia y la Unión Europea, a pesar de sus lazos históricos, no han conseguido privilegiar sus relaciones recíprocas. Además la Unión Europea y América Latina y el Caribe establecieron asociaciones estratégicas desde 1999, cuyo propósito es más que contribuir sosteniblemente sus relaciones proactivas, hecho que se lleva más de una década en estos encuentros entre los Jefes de Estado y de Gobierno con base a sus declaraciones birregionales y diversas reuniones frecuentes, no han sido suficiente que se logren compromisos concretos para una región como Latinoamérica que comparten principios y valores comunes (Cepal, 2015; Andi, 2015). Es por ello el interés de buscar espacios de convergencias y oportunidades entre los miembros 
de Unión Europea y Colombia que genere nuevos ámbitos comerciales, económicos y competentes, interesados en encontrar soluciones factibles, equitativas y sostenibles en aspectos como el medio ambiente y energético, cultural, educativa, tecnología y ciencia.

De manera superficial, vemos que la UE se podría llegar a complementar con Colombia, y así fue considerado por grandes analistas de la economía, debido a que el martes 26 de junio del año 2012 se logró firmar este importante acuerdo comercial para ambas economías.

En su momento, esta firma significo un gran avance y un suceso transcendental a nivel comercial y a largo plazo, fue uno de los momentos más importantes de la agenda política del país.

Nuestro país se decidió a firmar este "importante y significativo negocio", porque para la economía de nuestra región era más que necesario internacionalizar las operaciones mercantiles para que a su vez estás crezcan y se abran paso a otras economías mayores organizadas y de mayor auge.

Los sectores presupuestados a tener mayores beneficios a corto plazo son el sector del banano, las hortalizas, las frutas, el café, el aceite de palma, entre otros; también se escuchaba hablar muy seguido y positivamente del comercio interindustrial, es decir, del comercio de maquinaria, perfumería, plásticos, entre otros.

A simple vista, el TLC con la UE es una de las mejores opciones que tiene la economía del país para expandirse a otras regiones a nivel mundial. ¿Pero, realmente, el TLC es lo más conveniente para la economía colombiana? La respuesta a la pregunta va mucho más allá de un simple si o de un no. Es cierto que el TLC era considerado como la llave maestra que abriría esa puerta al comercio internacional, ese tiquete a una tasa preferencial baja (Reina y Oviedo, 
2011), en fin , pero hay que considerar que al hablar de la UE estamos hablando de varios "estados organizados" y que juntos buscan un camino para beneficiarse en conjunto; debemos reconocer que estamos compitiendo, por así decirlo, o interactuando con varios países que también buscan expandir su comercio y su tecnificación a bajo costo; y esto también es buscado por nuestros comerciantes, por eso, tal vez, estamos tomando un poco de riesgo al firmar este tipo de acuerdos.

Por otra parte, los productos colombianos (tal vez por nuestra cultura tan diversificada) tienen una demanda considerable a nivel internacional y casi siempre están exhibidos y son ofrecidos por un precio accesible al consumidor, esto nos indica que podemos diversificar aún más el mercado al que van nuestros productos, pero competir con el país dueño de ese mercado, como este caso, es una situación bastante difícil e incómoda de sobrellevar porque dar la pelea es diferente a conquistar un mercado en todo el sentido de la palabra.

\section{Conclusiones.}

EI TLC no es del todo malo, como algunos escépticos pudieron plantear, pero tampoco es la panacea, ambas naciones se vieron favorecidas en el inicio del negocio; en el año 2010 los intercambios comerciales entre Colombia y la UE fueron relativamente positivos para la UE, mientras que en los años siguientes (2011 y 2012) la balanza comercial se inclinó positivamente para Colombia; veamos:

En el 2010 Colombia vendió a la UE 4976 millones de dólares y le compro 5413 millones de dólares, lo que favoreció a la UE con 437 millones de dólares; por su parte, en los 12 meses del año 2011, el panorama giro drásticamente, pues, Colombia exporto 8868 millones de dólares a la UE, mientras que esta nos vendió 
productos por un valor superior a los 7000 millones de dólares; en el 2012 siguieron los resultados similares, vendiendo Colombia al bloque económico la suma de 8915 millones de dólares frente a los 7301 millones de dólares que vendió la UE.(Andi, 2015)

No se debe obviar que países como España y Alemania han tenido una fuerte actividad comercial con Colombia, los intercambios económicos cada día son más frecuentes y para el año 2012 totalizaron la no despreciable suma de 2692 y 2608 millones de dólares, respectivamente.

Los productos más comprados por Colombia a la UE durante el 2011 y 2012 (años definitivamente cruciales en la historia económica de ambos países) fueron maquinaria, los aparatos electrónicos relacionados con la industria aeronáutica, productos farmacéuticos, bienes del sector automotriz, productos químicos, plásticos, papel y cartón; en contraste, los bienes colombianos más comprados por la UE fueron el petróleo y sus derivados, además de las frutas y el café.(Andi, 2015)

En la actualidad, varios de nuestros productos han mejorado sus oportunidades de exportación, entre esos están incluidas las frutas, las hortalizas, los dulces y edulcorantes y en cuanto a los licores son lo que más han incrementado significativa y notoriamente su internacionalización.

En general, el TLC desde sus inicios, es decir, desde que fue firmado y hasta el presente ha brindado a gran parte de los sectores que componen nuestra economía una oportunidad importante para aumentar los niveles de crecimiento que hasta el momento se pueden calificar como productivos, nuestros productos tienen una demanda considerable que se debe saber aprovechar, además gozan de una buena reputación en lo que respecta a la calidad ofrecida, ofrecemos 
productos que a nivel internacional no serían tan accesibles de conseguir sino fueran por estos acuerdos.

Colombia es un país multifacético, geográficamente hablando, un país rico en minerales e hidrocarburos, goza de un territorio extenso que se compone de varias regiones naturales.

Las regiones naturales de Colombia son las divisiones territoriales basadas en características heterogéneas; estas divisiones y diferencias regionales están definidas básicamente por diversos factores como el suelo, sus usos y sus condiciones, además de la cercanía al mar de la zona, la frecuencia de lluvia, las condiciones del relieve (si es plano, montañosos o rocoso), entre muchas más.

En el caso de la región caribe, es el área marítima que ha cumplido uno de los papeles más importante del país a lo largo de toda su historia económica, está ubicada en el norte de Colombia, y es una privilegiada, pues su estratégica posición geográfica ha permitido, a lo largo de los años, que se den las condiciones adecuadas para los negocios comerciales con el exterior, físicamente la región está constituida por una llanura continental, limita al norte con el mar caribe (al cual debe su nombre), al sur con la región andina, al oriente con Venezuela y al occidente con el mar caribe y Panamá, además de estar bañada por el rio Magdalena, el Sinú (Córdoba), el San Jorge (Córdoba) y de varias ciénagas y lagunas.

La región caribe es favorablemente plana por lo que goza de cierta diversidad ecológica y climática que la hace única, la variedad de especies animales que en ella se encuentran es incalculable. Como si fuera poco, a la región pertenecen dos de las principales ciudades del país (Barranquilla y Cartagena), las cuales han sido pieza clave en la internacionalización global y comercial que el país ha estado en búsqueda en los últimos años: 


\section{Referencias.}

Andi. (2015). Inversión Extranjera. 2015, de ANDI Sitio web: http://www.andi.com.co/es/GAl/Guilnv/ActExt/InvExt/Paginas/Inc.aspx

Beethoven Herrera Valencia. (2011). TLC Colombia-Unión Europea: retos y desafíos. Diciembre 2011, de Friedrich Ebert Stiftung en Colombia FESCOL. Sitio web: http://library.fes.de/pdf-iles/bueros/kolumbien/08860.pdf

Carvajal Martínez, Jorge Enrique, \& Guzmán Rincón, Andrés Mauricio. (2017). Economía de mercado y democracia: elementos para una crítica al discurso del desarrollo promovido por las Instituciones Financieras Internacionales. Justicia, (31), 116-134. https://dx.doi.org/10.17081/just.22.31.2602

Cialti, Pierre-Henri. (2016). La mayor representatividad sindical en España*. Justicia, (30), 17-31. https://dx.doi.org/10.17081/just.21.30.1347

Comisión económica para América Latina y el Caribe (CEPAL). (2015). Inversión extranjera directa de la Unión Europea en América Latina y el Caribe. 2015, de Comisión económica para América Latina y el Caribe (CEPAL) Sitio web: http://www.cepal.org/prensa/noticias/comunicados/3/46573/hoja_informativa _ied_ue_es.pdf

Cárdenas, L. (2013). Prácticas de lectura y escritura en la universidad. ¿Qué y cómo leen y escriben los estudiantes de licenciaturas? Revista Amauta, 21, 139-162

Cruz, E. (2015). la Ciudadanía ECológiCa En aBya-yala/ amériCa latina. Amauta, 87-103. 
Damm, Arturo. (2008). Ventajas de la inversión extranjera. Marzo 04 2008, de elcato.org Sitio web: http://www.elcato.org/ventajas-de-la-inversionextranjera

María Wilhelmina, Joseph Antonia Van Gool, Miriam García Ferrer, Ivo Hoefkens, Sergio Díaz-Granados, Calos Andrés De Hart Pinto, Gabriel Duque Mildenberg. (2012). Acuerdo Comercial Colombia Unión Europea. mayo 2012, de Ministerio de comercio industria y turismo Sitio web: http://www.eeas.europa.eu/delegations/colombia/documents/page_content/c olombia_unio_n_europea_acuerdo_comercial_jul_11_heavy_es.pdf

Mauricio Reina y Sandra Oviedo. (2011). Colombia y el TLC con la Unión Europea. Julio 2011, de Friedrich Ebert Stiftung en Colombia Fescol Sitio web: http://library.fes.de/pdf-files/bueros/kolumbien/08401.pdf

Mejía Cáez, Miguel Ramón. (2017). El derecho internacional de los derechos humanos, un nuevo concepto. Justicia, (32), 38-63. https://dx.doi.org/10.17081/just.23.32.2904

Michael Jetter. (2011). La lucha de Estados Unidos y la Unión Europea: implicaciones para Colombia. Octubre 19 2011, de Dineromentor.com Sitio web: http://www.dinero.com/opinion/opinion-on-line/articulo/la-luchaestados-unidos-union-europea-implicaciones-para-colombia/137799

Ministerio de Comercio Industria y Turismo. (2016). ABC del Acuerdo Comercial con la Unión Europea. Junio 05 2013, de Ministerio de Comercio Industria y Turismo Sitio web: http://www.mincit.gov.co/publicaciones.php?id=3406 
Mónica Vargas y Tom Kucharz. (2010). Tratados de Libre Comercio entre la Unión Europea y América Latina. Mayo 09 2010, de Revista Soberanía Alimentaria Sitio web:

https://revistasoberaniaalimentaria.wordpress.com/2010/05/09/tratados-delibre-comercio-entre-la-union-europea-y-america-latina/

Óscar Güesguán Serpa. (2015). Un acuerdo que empezó con pie derecho. Marzo 07 2015, de El Espectador Sitio web:

http://www.elespectador.com/noticias/economia/un-acuerdo-empezo-piederecho-articulo-547932

Pabón Arrieta, Juan Antonio, \& Torres Argüelles, Alfredo. (2017). La interpretación jurídica en el litigio estratégico en defensa de los derechos humanos en Colombia. Justicia, (32), 227-242. https://dx.doi.org/10.17081/just.22.32.2915

Rodríguez-Burgos, Karla, Martínez Cárdenas, Alán Alejandro, \& Rodríguez-Serpa, Ferney Asdrual. (2017). Estudio empírico sobre los valores democráticos de tolerancia y respeto en la generación milenaria. Justicia, (31), 135-150. https://dx.doi.org/10.17081/just.22.31.2603

Sanabria, Fabián. (2016). Estado, instituciones democráticas y postconflicto en Colombia*. Justicia, (30), 86-95. https://dx.doi.org/10.17081/just.21.30.1351

Vanguardia.com. (2012). Riesgos y beneficios del TLC con la Unión Europea. Diciembre 12 2012, de COLPRENSA Sitio web: http://www.vanguardia.com/economia/nacional/187223-riesgos-y-beneficiosdel-tlc-con-la-union-europea 